\title{
Training oncoplastic breast surgeons: the Canadian fellowship experience
}

\author{
J. Maxwell MD, ${ }^{*}$ A. Arnaout $\mathrm{MD} \mathrm{MSc}_{1}^{\dagger}$ R. Hanrahan $\mathrm{MD}_{1}^{\ddagger}$ and M. Brackstone $\mathrm{MD} \mathrm{MSc} \mathrm{PhD}^{\S}$
}

\section{ABSTRACT}

Background Oncoplastic breast surgery combines traditional oncologic breast conservation with plastic surgery techniques to achieve improved aesthetic and quality-of-life outcomes without sacrificing oncologic safety. Clinical uptake and training remain limited in the Canadian surgical system. In the present article, we detail the current state of oncoplastic surgery (ops) training in Canada, the United States, and worldwide, as well as the experience of a Canadian clinical fellow in ops.

Methods The clinical fellow undertook a 9-month audit of breast surgical cases. All cases performed during the fellow's ops fellowship were included. The fellowship ran from October 2015 to June 2016.

Results During the 9 months of the fellowship, 67 mastectomies were completed (30 simple, 17 modified radical, 12 skin-sparing, and 8 nipple-sparing). The fellow participated in 13 breast reconstructions. Of 126 lumpectomies completed, 79 incorporated oncoplastic techniques.

Conclusions The experience of the most recent ops clinical fellow suggests that Canadian ops training is feasible and achievable. Commentary on the current state of Canadian ops training suggests areas for improvement. Oncoplastic surgery is an important skill for breast surgical oncologists, and access to training should be improved for Canadian surgeons.

Key Words Oncoplastic breast surgery, breast conservation, surgical education, breast reconstruction, clinical fellowships, breast cancer

Curr Oncol. 2017 Oct;24(5):e394-e402

www.current-oncology.com

\section{INTRODUCTION}

Breast cancer is the most common cancer affecting Canadian women, and the 2nd leading cause of female cancer deaths. It is estimated that more than 25,000 women are diagnosed with breast cancer annually ${ }^{1}$. The evolving nature of breast cancer care requires that the management of breast disease be an important part of surgical education programs from residency through fellowship.

In a 2012 survey, graduating residents indicated that they were uncomfortable discussing the complexities of medical management of breast cancer care with patients ${ }^{2}$. Breast surgery rotations are often completed early in medical training, with senior residents performing minimal breast surgery and receiving less instruction in the comprehensive management of breast disease ${ }^{2}$.

Data suggest that outcomes are better for breastfocused surgeons than for general surgeons, and highvolume breast surgery is correlated with improved outcomes at the levels of both the hospital and the individual surgeon $^{3,4}$. Patient satisfaction is higher when care is handled by breast-focused surgeons ${ }^{5}$. With a growing number of clinicians dedicating their practice to breast disease, and given the advances in breast cancer care, the Society of Surgical Oncology (sso) began training fellows in recognized U.S. programs in 2004. Since then, the number of sso-approved breast fellowship programs has increased from 24 to $46^{6}$. The University of Toronto began its breast fellowship training program in 2002, accepting 1-2 fellows annually. Western University in London, Ontario, trained its first breast ablative and reconstructive surgery fellow in 2007 and has accepted trainees sporadically since then.

The most significant recent advance in surgical technique for breast-conserving surgery is oncoplastic surgery (ops). The aim of ops is to combine oncologic breast conservation methods with plastic surgery techniques to achieve improved aesthetic and quality-of-life outcomes without sacrificing oncologic safety. First developed in the 1980s in Europe, the ops approach has been refined over the decades, but clinical uptake-and consequently 
training-remains limited in the Canadian surgical system. A recent study highlighted lack of training as a major barrier to the use of ops in Canada ${ }^{7}$.

In this article, we detail the current state of ops training in Canada, the United States, and worldwide, as well as the experience of a Canadian clinical fellow in breast ops.

\section{OPS TRAINING BY JURISDICTION}

\section{Canadian OPS Training}

Currently, ops training is entirely separate from training for breast surgical oncology and for general surgical oncology. A single formal breast surgical oncology fellowship is available in Canada. That 1-year program is offered by the University of Toronto, which accepts 1-2 fellows annually. Training occurs at two major sites: the Sunnybrook Health Sciences Centre and the University Health Network. The program exists outside the sso match and is not accredited by the Accreditation Council for Graduate Medical Education or the Royal College of Physicians and Surgeons of Canada. Having been in existence for more than a decade, the program has trained many of Canada's current breast surgeons. It is separate from the general surgical oncology fellowship and offers rotations in medical and radiation oncology, radiology, pathology, and plastic surgery in addition to breast surgical oncology. No formal oncoplastic training is built into the curriculum. Fellows do spend time with local plastic surgeons learning about breast reconstruction; however, the goal of that rotation is not to train breast oncology fellows to perform independent breast reconstruction or oncoplasty. The fellowship offers a high volume of breast surgical oncology cases, plus the opportunity to gain expertise in the multidisciplinary management of breast disease.

Less-formal breast surgical oncology fellowship opportunities are available across the country, but depend largely on the clinical fellow for initiation and set-up. Many of the programs accept clinical fellows on an intermittent basis. Additional formalized breast surgery fellowship programs are in development, but have yet to accept clinical fellows.

Multiple general surgical oncology fellowships are offered across Canada. Those programs admit fellows through a formal matching service (usually the sso match) and offer broad general surgical oncology training, with specific breast surgery rotations, but no formal ops component.

Canadian surgeons currently performing ops have generally obtained their skills through courses taken internationally on their own time or through the additional formal oncoplastic fellowships discussed in the subsections that follow.

\section{U.S. OPS Training}

Breast surgical oncology training in the United States was formalized in 2003 through the joint efforts of the sso, the American Society of Breast Disease, and the American Society of Breast Surgeons. The first clinical fellows began training in 2004. A survey of early breast fellowship graduates (2005-2009) demonstrated that only 53\% felt well-prepared to perform oncoplastic breast surgery ${ }^{6}$.
In recent years, an increasing number of sso-approved breast fellowship programs have offered some level of ops training. In 2016, 11 of 46 sso-approved breast fellowships expressly mentioned ops in their program overviews; all programs offer a plastic or reconstructive surgery rotation ${ }^{8}$.

Breast surgery fellowships outside the sso match are available; however, information about their access to oncoplastic training is limited. As with the less-formal Canadian fellowships, those programs depend on the clinical fellow for initiation and set-up.

\section{International OPS Training}

Internationally, the need for increased ops training and resources has been widely recognized.

In 2007, the British Association of Surgical Oncology, in conjunction with the British Association of Plastic, Reconstructive and Aesthetic Surgeons and the Training Interface Group in Breast Surgery, produced a breast ops guide to good practice. The guide established the importance of breast ops and set forth mandates for essential skills in ops, as well as a framework for education and training 9 .

The 7th Portuguese Senology Congress focused on the provision of ops and the availability of training in various countries ${ }^{10}$. In Portugal, specialty breast training is in a reorganizational phase, with plans for the development of a breast-specific curriculum, with formal examination for general and plastic surgery trainees and for gynecologists.

A Spanish consensus statement mandating that ops be offered to breast care patients has led to the ongoing development of training programs in that country ${ }^{10}$.

Brazil has a significant history of progress in plastic surgery. That progress is reflected in the current state of Brazilian ops, wherein plastic surgeons are the predominant providers, covering $75 \%$ of ops services in the country $^{10}$. In 2009, an oncoplastic training centre was established with the approval of the Brazilian Society of Mastology. The training centre has offered a 21-module course that takes place on one weekend per month and that targets practicing breast surgeons rather than recent surgical graduates ${ }^{11}$.

The United Kingdom has long been a forerunner in the field of breast ops. Formal ops training has been available to appropriate candidates since $2002^{12}$. Candidates must complete 6 years of general training, with ops education included in the final 3 years ${ }^{13}$. That training can be supplemented with an additional year of senior-level oncoplastic training in Training Interface Group positions. Trainees are accepted from both general and plastic surgery backgrounds. There are 9 available spots in total, and acceptance into the programs is highly competitive ${ }^{13}$.

Oncoplastic training is well defined in France. Trainees enter their fellowship programs after 5 years of obstetrics and gynecology training. Fellowships are tailored to the individual trainee, with 3 years' experience in gynecology, breast surgical oncology, and plastics followed by 1 year of ops training certification (Kaufman G. Oncoplastic surgery training: European fusion model versus American 2-step. Presented at: American Society of Breast Surgeons 16th Annual Meeting; Orlando, Florida; 29 April-3 May 2015).

In Australia and New Zealand, access to ops training is highly variable, with little infrastructure for formal 
ops education. The Breast Surgeons of Australia and New Zealand is working to formalize post-fellowship training in breast surgery and has formed an oncoplastic subcommittee to monitor ops training. Breast units are being accredited as formal training centres, and a centralized application program is in progress ${ }^{14,15}$.

\section{A CANADIAN FELLOWSHIP EXPERIENCE}

Western University has offered an ablative and reconstructive breast surgery fellowship intermittently since 2007. Two Canadian fellows have successfully completed the program, which has also accepted and trained international fellows. To reach the necessary case volume, a clinical fellow trains at three separate sites for 3-4 months at a time. Case volume becomes a challenge because many lumpectomies can use basic ops techniques, which require minimal, if any, additional training. A smaller subset of patients are candidates for higher-level ops procedures.

Given the urgency of oncologic surgery, cases cannot be bundled for fellows, leading to a training challenge and necessitating multiple training sites. The Western University fellowship has therefore coordinated efforts with oncoplastic surgeons at two other large-volume breast programs in Ontario. Those programs are The Ottawa Hospital's Women's Breast Health Centre (based at the University of Ottawa) and the Simcoe Muskoka Regional Cancer Program (based at the Royal Victoria Regional Health Centre, a high-volume community hospital). Each site has access to a regional cancer centre and a full complement of multidisciplinary breast cancer care, including medical and radiation oncology, radiology, pathology, and plastic surgery.

At each site, the fellow worked under the supervision of breast surgical oncologists with additional training and expertise in breast ops. Oncoplastic and reconstructive procedures were completed, together with traditional mastectomy and breast conservation. The clinical fellow was also trained by plastic surgeons in methods of immediate and delayed breast reconstruction and reduction mammoplasty level II oncoplastic techniques. Operative experience was supplemented by attendance at weekly multidisciplinary rounds.

The fellow attended 2-3 breast surgical oncology clinics weekly. Clinics represented a mix of new patients with benign or malignant disease and follow-up patients. Patients were generally not seen in multidisciplinary clinics or in conjunction with plastic surgery. Grand rounds on breast ops were presented by the fellow. Additionally, ops lectures were organized and presented by the fellow to surgeons at outside institutions. Rotations in medical and radiation oncology, radiology, and pathology are offered to fellows who have not previously completed a breast surgical oncology fellowship.

Research was encouraged throughout the fellowship. No formal research time was incorporated into the schedule; however, ample time was available. Research funding was grant-dependent. The fellow attended two conferences during the year: the sso annual meeting and the annual meeting of the American Society of Breast
Surgeons. Costs for those meetings were the responsibility of the fellow.

At each site, the fellow interacted with on-service general surgery residents and medical students. Residents were at the PGY3 level or higher. The fellow was involved in resident teaching in both the operating room and the clinic.

The fellowship was self-funded; that is, the clinical fellow did not receive a salary from Western University or any of the hospitals affiliated with the program. The fellow billed the provincial health insurance plan as a surgical assistant for each operative procedure completed.

No general surgery call was required during this fellowship. The option to take call was available. On occasion, the fellow covered call in conjunction with their attending surgeon.

Feedback was informal and unscheduled, but given routinely. No formal evaluations took place. No examination was required upon fellowship completion. This fellowship was not accredited by the Accreditation Council for Graduate Medical Education or the Royal College of Physicians and Surgeons of Canada. That lack of accreditation is not unique to this specific program; at the time, no Canadian or U.S. breast surgery fellowships were accredited by the Accreditation Council for Graduate Medical Education or the Royal College of Physicians and Surgeons of Canada.

Graduating fellows are expected to independently perform level I and II ops, plus implant-based immediate and delayed breast reconstruction.

Recently, The Ottawa Hospital's Women's Breast Health Centre formalized its own 1- to 2-year oncoplastic fellowship training program to meet the increasing demand for ops. Recent hiring of 3 young oncoplastic breast surgeons and 2 plastic surgeons interested in training general surgeons in oncoplastic techniques has allowed Ottawa to independently offer the oncoplastic case volume required to adequately train a fellow over a span of up to 2 years. The fellowship, which is co-directed by a plastic surgeon and a breast surgeon, is the culmination of work on a dedicated oncoplastic curriculum and represents a collaboration between general and plastic surgeons. Formal oncoplastic fellowship objectives encompass both level I and II volume displacement techniques, with exposure to implant- and tissue-based volume replacement reconstruction.

The fellow can tailor the program to meet the demands of a future academic or community position, because a graduate (MSc) program can be built into the fellowship if desired. At the end of the fellowship, it is expected that the fellow will have acquired the skills to perform the full range of oncoplastic techniques (levels I and II) independently. However, as much training experience as desired in performing various aspects of breast reconstruction can also be added-for example, immediate prosthetic or autologous reconstruction as trained by the plastic surgeons involved in the fellowship. Candidates accepted into the program are also involved in community outreach, continuing medical education, and research in ops.

The first fellow was accepted for training in July 2017. Eligible applicants are general surgery graduates (new or already established in practice) and graduates who have already completed a breast or general surgical oncology fellowship, but who wish to gain more experience in ops. 


\section{RESULTS}

The clinical fellow undertook a 9-month audit of breast surgical cases, which included all cases performed during the fellow's ops fellowship (October 2015 to June 2016). Personal case logs were used for documentation.

Over the 9 months of the fellowship, 67 mastectomies were completed: 30 simple, 17 modified radical, 12 skinsparing, and 8 nipple-sparing mastectomies. The fellow participated in 10 cases of expander- or implant-based reconstruction and 3 deep inferior epigastric perforator reconstructions (Table I).

Of the 126 lumpectomies completed, 79 incorporated oncoplastic techniques, including reduction, round block, racquet, batwing, and V or J mammoplasty. Table II sets out a complete list of breast-conserving and oncoplastic cases. The ops cases are divided into level I and level II procedures, per the classification system proposed by Clough and colleagues $^{16}$ (Table III). Level I procedures are less complex and do not require additional ops or plastic surgery training; level II procedures are of increasing complexity and require additional training or plastic surgery involvement (see Table III).

\section{DISCUSSION AND RECOMMENDATIONS}

It is evident from the case logs that the fellowship program offered a wide variety of traditional, ops, and reconstructive breast surgery experience. The case volume was excellent and comparable to that in traditional breast surgical oncology fellowships. The program benefited from the collaboration and expertise of surgeons around the province, without whom it would have been impossible to obtain adequate ops volumes.

The length of the fellowship was tailored to allow the fellow to begin employment after 9 months; typically, it would be 1 year in duration. Proficiency in both level $\mathrm{I}$ and level II ops was reached over the allocated time. In both the United States and the United Kingdom, ops fellowships have adopted a 1-year timeframe, further substantiating the current duration of the Western University program. The 2007 guidelines from the European Society of Breast Cancer Specialists outline the minimum experience required to successfully complete an oncoplastics fellowship $^{17}$. With respect to ops, the recommendation is that the trainee have assisted or observed at 10 and personally have performed 5 breast remodelling procedures after breastconserving surgery. The numbers achieved in 9 months of the Western University fellowship greatly exceeded that suggested volume.

Although a recent survey demonstrated that $47.6 \%$ of Ontario academic breast surgeons incorporate some level of ops into breast conservation, most of those surgeons did not have additional subspecialty training in ops ${ }^{7}$. It can be extrapolated that, without additional training, those surgeons are generally performing level I ops. For that reason, it was necessary for the fellowship to include work with ops experts at three separate sites, providing maximal exposure to both level I and level II ops. The fellow spent 3 months at each site. Sites were, on average, 3-6 driving hours apart, making a daily commute impossible. Centralizing the
TABLE I Summary of fellowship mastectomy and reconstruction cases, October 2015-June 2016

\begin{tabular}{lc}
\multicolumn{1}{c}{ Surgical procedure } & Performed \\
\hline Mastectomy $(n)$ & \\
Simple & 30 \\
Modified radical & 17 \\
Skin-sparing & 12 \\
Nipple-sparing & 8 \\
Reconstruction $(n)$ & \\
Tissue expander or implant & 10 \\
Deep inferior epigastric perforator & 3 \\
Other autologous flap & 0 \\
\hline
\end{tabular}

TABLE II Summary of fellowship lumpectomy and oncoplastic surgery (OPS) cases, October 2015-June 2016

\begin{tabular}{lcc}
\hline \multicolumn{1}{c}{ Surgical procedure } & $\begin{array}{c}\text { Performed } \\
(\boldsymbol{n})\end{array}$ & $\begin{array}{c}\text { OPS } \\
\text { level }^{\mathbf{a}}\end{array}$ \\
\hline Traditional lumpectomy & 47 & $\mathrm{NA}$ \\
$\begin{array}{l}\text { Oncoplastic lumpectomy } \\
\text { Simple glandular reapproximation }\end{array}$ & 53 & $\mathrm{I}$ \\
Round block & 3 & $\mathrm{II}$ \\
Batwing or hemi-batwing & 1 & $\mathrm{I}$ \\
Racquet & 5 & II \\
Reduction mammoplasty & 16 & $\mathrm{II}$ \\
V- or J-Mammoplasty & 1 & $\mathrm{I}$ \\
\hline
\end{tabular}

a See Table III.

NA = not applicable.

fellowship is a goal that will be achieved only when availability of ops surgeons is more widespread.

One criticism of the program is the lack of formal objectives and evaluation. To remedy that situation, the most recent clinical fellow, together with the program director, was heavily involved in the formalization of fellowship objectives at the university level, allowing for more appropriate evaluation and feedback for future fellows. Recently, departmental leads for the ops fellowship have been assigned from surgical oncology, plastic surgery, radiology, medical and radiation oncology, and pathology. A formal set of objectives spanning both surgical and off-service rotations has been developed with input from each departmental lead. Those objectives incorporate the canmeds roles set out by the Royal College of Physicians and Surgeons of Canada (see Table Iv).

The development of formal fellowship objectives from each department has raised questions about the nature of the program itself. Is this a breast surgical oncology fellowship with an ops focus? Or is it solely a surgical fellowship? The distinction is important. The most recent fellow had previously completed a formal breast surgical oncology fellowship and was comfortable forgoing off-service breast oncology rotations to focus on surgical technique. That need is assessed for each incoming fellow. For fellows 
TABLE III Level I compared with level II oncoplastic surgery techniques

\begin{tabular}{|c|c|c|}
\hline & Level I & Level II \\
\hline ! & Reasonable to consider without plastic surgery or additional training & Requires plastic surgery support or additional training \\
\hline & Less than $20 \%$ breast volume loss & Breast volume loss of $20 \%-50 \%$ \\
\hline & Use in $90 \%$ of cases & Excision of skin to reshape breast \\
\hline & Avoid in fatty breasts & Recentralization of nipple areolar complex \\
\hline & & Based on mammoplasty techniques \\
\hline & & Less used \\
\hline & & - Safer in fatty breasts \\
\hline
\end{tabular}

without prior breast surgical oncology training, rotations in that area are available. But then the issue of case volume arises: If the program were to abandon some of its focus on ops in favour of formal breast surgical oncology training, would the fellow achieve enough experience in ops technique to become proficient in 1 year? Per the guidelines from the European Society of Breast Cancer Specialists, even with the incorporation of additional off-service rotations, the fellow would likely reach the required number of ops cases in 1 year.

Another issue to consider is the addition of a year of education to the already lengthy Canadian surgical training experience. That extra time might, in itself, discourage applicants. It is recommended that, to fully understand the complexities of breast cancer management, future applicants consider completing a formal breast surgical oncology fellowship before taking on this ablative and reconstructive fellowship.

It should also be mentioned that collaboration with plastic surgeons is imperative for the fellowship to function. The fellow was generally well accepted by the plastic surgery community at each site; however, the realm of ops remains a territorial issue. The fellowship experience would have benefited from more buy-in from plastic surgeons. For both general and plastic surgeons, ops requires a shift in thinking. Traditionally, general surgeons or surgical oncologists have performed the ablative operation in Canada, and all reconstruction has been left to the plastic surgery team. To date, that approach has also included post-lumpectomy tissue rearrangements, effectively making plastic surgeons the sole providers and caretakers of cosmesis. Surgeons and surgical oncologists must concern themselves more with aesthetics (without sacrificing cancer care), and plastic surgeons must not view ops as diminishing their role in breast reconstruction. The ops function supplements their role and increases the amount of breast conservation being performed, while freeing time for plastic surgeons to focus on whole-breast reconstruction.

Funding for the fellowship remains an issue. As it stands, the fellow is responsible for conference, housing, and transportation costs across all sites. However, the fellow does have the ability to cover general surgery call and to assist in outside procedures to supplement income. Grant applications are encouraged to further support the fellowship. As the program continues to grow, a new funding model could be considered. In other breast fellowship programs, the clinical fellows bill the provincial government as surgical assists. All funds are directed to a common pool, and salaries for the fellows are paid from the pool-an approach that allows for payment to continue during off-service rotations. That model was established over the years and initially required each fellow to have grant funding to be admitted, providing start-up funds until the common pool was created. That model might be one to consider in the future for the Western University fellowship, especially if off-service rotations are introduced.

Despite the issues raised in this discussion, the fellowship experience described here clearly demonstrates that adequate ops volumes and training are achievable within the Canadian system. Training programs for ops can benefit the local population by increasing access to a variety of surgical options. As more fellows are trained and disperse across the country, patient access to ops will increase on a larger scale, thus providing a potential solution to the scheduling issues and geographic inequalities that arise when a centre does not have access to plastic surgery ${ }^{13}$.

\section{CONCLUSIONS}

The experience of the most recent ops clinical fellow suggests that, although some adjustments are required to maintain a long-term ops fellowship program, Canadian ops training is feasible and achievable. Much of breast cancer care has shifted focus to survivorship. The use of ops allows for less-invasive oncologic surgery, with fewer potential complications than are encountered with mastectomy and reconstruction, and with appropriate esthetic results. The resulting improvements in body image, psychosocial outcomes, and comfort with intimacy all contribute to improved quality of life ${ }^{18-20}$. For breast surgical oncologists, ops is an important skill, and access to training should be improved for Canadian surgeons.

Finally, it should be noted that the opinions, suggestions, and recommendations in this article are solely those of the authors and were not reviewed or approved by any Canadian surgical governing body.

\section{CONFLICT OF INTEREST DISCLOSURES}

We have read and understood Current Oncology's policy on disclosing conflicts of interest, and we declare that we have none.

\section{AUTHOR AFFILIATIONS}

*Division of Surgical Oncology, University of Nebraska Medical Center, Omaha, NE, U.S.A.; ${ }^{\dagger}$ Division of Surgical Oncology, The 
TRAINING ONCOPLASTIC BREAST SURGEONS, Maxwell et al.

TABLE IV Combined breast surgical oncology and oncoplastic fellowship rotational objectives with CanMEDS Roles

\section{Leader}

1. Breast imaging

1.1 Understand the techniques of diagnostic mammography, including the BI-RADS lexicon recommendations for additional views; and identify mammographic characteristics of benign, probably benign, and malignant disease.

1.2 Understand the techniques of breast sonography and distinguish normal breast sonographic anatomy, sonographic characteristics of simple cysts, complex cysts, complicated cysts, well-circumscribed probably benign mass, and solid mass of suspicious nature.

1.3 Demonstrate experience in selecting image-guided breast intervention procedures, including, but not limited to, ductograms, image-guided (that is, ultrasound, stereotactic, MRI, and others) fine-needle aspiration, and core and vacuum-assisted biopsies.

1.4 Demonstrate understanding of preoperative localization procedures using wire and radioactive seeds.

1.5 Discuss the evolving breast imaging technologies.

1.6 Evaluate the present indications for and possible future applications of MRI in the management of malignant and benign breast disease.

1.7 Select, recommend, and interpret the techniques of breast lymphoscintigraphy.

1.8 Discuss the complexities, advantages, and disadvantages of breast screening trials in women at different age groups.

1.9 Understand the concept of oncoplastic breast surgery with respect to preoperative and postoperative (surveillance) imaging.

2. Breast surgery (London, Barrie, and Ottawa rotations)

2.1 Evaluate and manage common benign and malignant breast conditions.

2.2 Assess the indications and contraindications for, and demonstrate experience in the performance and interpretation of, the results of common in-office procedures, including but not limited to cyst aspiration, fine-needle aspiration, percutaneous core biopsy with and without image guidance, punch biopsy of skin.

2.3 Assess the indications for techniques to optimize cosmetic outcome, minimize surgical trauma, and achieve the best oncologic outcome for cancer operations for all major breast procedures, including but not limited to breast biopsy, wire and seed localization biopsy, duct excision, lumpectomy, simple mastectomy, and modified radical mastectomy with or without skin or nipple sparing, chest wall resection, axillary lymph node dissection, and sentinel lymph node mapping. This includes oncoplastic procedures in breast-conserving cases. The surgical breast fellow must demonstrate proficiency in the performance of these procedures.

2.4 Oncoplastic breast surgery specific objectives

i. Understand the principles of oncoplastic surgery, with identification of suitable patients based on patient and disease characteristics for oncoplastic breast-conserving procedures.

ii. Understand the types of incisions and methods of closure for level I/II procedures and pedicle flaps for level II procedures.

iii. Develop an approach to preoperative marking for level II oncoplastic procedures.

iv. Understand the blood supply to the nipple and the postoperative complications/imaging sequelae that can arise in oncoplastic procedures.

2.5 Demonstrate proficiency in interdisciplinary evaluation and presurgical treatment planning with multiple disciplines, including but not limited to, radiology, plastic and reconstructive surgery, medical oncology, radiation oncology, genetics, and pathology.

2.6 Identify the indications for and techniques of palliative surgical procedures for locoregional relapse as well as metastatic foci.

2.7 Evaluate and manage arm lymphedema as a side effect of breast cancer treatment.

2.8 Explain evolving surgical technologies such as percutaneous ablation, core vacuum resection, focused ultrasound, ductal lavage, and ductoscopy.

3. Genetics

3.1 Identify patients at high risk for developing breast cancer, including risk factors such as pathologic, familial, genetic, and previous cancer-inducing therapies (that is, childhood radiation).

3.2 Discuss the epidemiologic evidence of the effect of environmental factors (broadly defined as nutrition, lifestyle, pollutants, chemicals, social economic status, etc.) on high-risk patients.

3.3 Advise patients regarding estimations of risk by contemporary models and risk reduction by screening, medication, and surgery.

3.4 Review the available clinical trials for breast cancer risk reduction and facilitate the option of participation in such trials.

3.5 Advise patients regarding indications, usefulness, costs, complications, and privacy issues of genetic testing.

3.6 Take a detailed family pedigree and history.

3.7 Interpret the various pathology findings as they influence risk.

3.8 Describe and evaluate options for breast-conserving therapy in patients suspected of an inherited susceptibility.

3.9 Identify resources available for genetic testing and counselling. 
TABLE IV Continued

Leader (continued)

4. Community support or outreach, career development, and leadership training

4.1 Identify and contact local patient advocate organizations, and participate in relevant activities.

4.2 Identify ways to provide public service to the community.

4.3 Promote the best standard of breast care and screening.

4.4 Identify hospital or regional cancer program-based support systems, and participate in relevant activities.

4.5 Participation in courses to enhance leadership potential in breast surgical oncology.

4.6 Leadership of regional multidisciplinary rounds and participation in province-wide interdisciplinary continuing medical education activities.

4.7 Mentorship of future trainees for breast surgical oncology career.

5. Psychooncology

5.1 Recognize needs for support for patients and their families throughout diagnosis, treatment, transition to surveillance, and relapse.

5.2 Recognize and respect cultural diversity and the different needs of patients and their families with regard to illness and treatment.

5.3 Exhibit a sensitive and culturally appropriate style of communicating with patients and their families.

5.4 Explain and discuss all aspects of care with patient in lay terms.

5.5 Recognize patients at high psychosocial risk and identify resources for referral.

5.6 Participate in existing local support groups.

5.7 Discuss complementary therapies or integrated care.

6. Radiation oncology

6.1 Describe the radiation oncology process to the patient, including simulation, treatment planning, treatment delivery, and acute and chronic effects of therapy.

6.2 Assess the indications and contraindications of post-breast-conservation radiation therapy in both ductal carcinoma in situ and invasive carcinomas, as well as post-mastectomy radiation therapy, axillary radiation therapy, management of chest wall recurrences, and inclusion or exclusion of supraclavicular or internal mammary fields.

6.3 Discuss the role of radiation therapy in axillary nodal disease as it pertains to surgical decision-making.

6.4 Identify indications for and techniques of palliative radiation for locoregional recurrence and metastatic disease.

6.5 Discuss evolving the technology of more localized radiation techniques including accelerated partial breast irradiation and intraoperative radiation therapy.

6.6 Understand the effects of radiation as they relate to immediate and delayed breast reconstruction.

6.7 Understand the concept of oncoplastic breast surgery with respect to radiation planning.

7. Medical oncology

7.1 Assess the indications and contraindications for adjuvant systemic chemotherapy and hormonal therapies.

7.2 Describe the mechanism of action and the risks, benefits, and indications for existing and developing targeted therapies.

7.3 Describe the most commonly prescribed chemotherapy and hormonal agents and their associated acute and chronic toxicities.

7.4 Identify and manage toxicities of prescribed agents.

7.5 Identify the indications, techniques, and interdisciplinary coordination required for neoadjuvant and "sandwich" chemotherapy.

7.6 Demonstrate experience in the interdisciplinary management of recurrent and metastatic disease including palliative care.

7.7 Discuss the personalized approach to systemic therapy in the patient with metastatic disease.

7.8 Manage patient and familial needs for psychosocial support, intervention, hospice, and crisis management.

8. Pathology

8.1 Explain and evaluate the benign and malignant pathologic aspects of breast disease.

8.2 Understand optimal techniques for marking, processing and assessing the pathology specimen.

8.3 Identify special pathology issues pertinent to the treatment of breast cancer.

8.4 Achieve familiarity with the performance and assessment of immunohistochemistry for estrogen receptor, progesterone receptor, and HER2/neu.

8.5 Gain an understanding of fluorescent in situ hybridization testing for HER2/neu gene amplification, how it is assessed, and the clinical implications of the results of this testing.

8.6 Discuss evolving pathology technology.

8.7 Stage breast cancer clinically and pathologically. 
TABLE IV Continued

Leader (continued)

9. Plastic and reconstructive surgery

9.1 Understand the role and technical procedures of tissue expander, implant, and a variety of flap reconstruction techniques for immediate and delayed reconstruction in the setting of post-mastectomy reconstructive surgery.

9.2 Understand the role and technical procedures of reconstructive techniques in breast conservation and contralateral balancing procedures. The breast surgical fellow is expected to be proficient in these procedures.

9.3 Understand general breast plastic surgery procedures such as augmentation and reduction as they relate to total management of women with benign and malignant breast disease.

9.4 Explain and evaluate the interrelationship of adjuvant therapies on the planning and timing of plastic and reconstructive surgery.

Communicator

1. Obtain and synthesize a breast history, including risk factors for breast cancer, and signs and symptoms of disease.

2. Discuss the rationale for investigations, including imaging and biopsy.

3. Deliver bad news in a compassionate and sensitive manner, taking into account the patient's individual psychological and social needs.

4. Obtain informed consent, including an effective explanation of rationale for recommended surgical approach and its risks and benefits. Collaborator

1. Consult effectively with other physicians and health care professionals in the multidisciplinary cancer team.

2. Demonstrate knowledge about palliative care options available to patients and their families toward the end of the disease process.

3. Communicate effectively during interdisciplinary team activities, including multidisciplinary cancer conference.

Professional

1. Coordinate the multidisciplinary cancer conference and breast rounds, and document the decision-making process.

2. Critically appraise relevant current literature on the surgical management of breast cancer.

Health advocate

1. Identify and contact local patient advocate organizations and participate in relevant activities.

2. Identify ways to provide public service to the community.

3. Promote the best standard of breast care and screening.

4. Advocate on the patient's behalf for the best care and support throughout their diagnosis, treatment, and surveillance or relapse.

Scholar

1. Participate in clinical trial development and patient enrolment.

2. Participate in prospective and retrospective clinical research, including clinical trials.

3. Participate in enrolment of patients in available national protocols.

4. Participate in the conduct and critical review of research studies.

5. Participate in the preparation of manuscripts for publications in lay or professional journals.

$\mathrm{MRI}=$ magnetic resonance imaging .

Ottawa Hospital, Ottawa, ON; ${ }^{\ddagger}$ Department of Surgery, Royal Victoria Regional Health Centre, Barrie, ON; ${ }^{\S}$ Division of Surgical Oncology, Schulich School of Medicine and Dentistry, Western University, London, ON.

\section{REFERENCES}

1. Canadian Cancer Society. Breast Cancer Statistics [Web page]. Toronto, ON: Canadian Cancer Society; 2017. [Available at: http://www.cancer.ca/en/cancer-information/cancertype/breast/statistics/?region=on; cited 20 September 2016]

2. Wilson JP, Miller A, Edge SB. Breast education in general surgery residency. Am Surg 2012;78:42-5.

3. Pass HA, Klimberg SV, Copeland EM 3rd. Are "breast focused" surgeons more competent? Ann Surg Oncol 2008;15:953-5.

4. Peltoniemi P, Peltola M, Hakulinen HT, Hakkinen U, Pylkkanen L, Holli K. The effect of hospital volume on the outcome of breast cancer surgery. Ann Surg Oncol 2011;18:1684-90.

5. Waljee JF, Hawley S, Alderman AK, Morrow M, Katz SJ. Patient satisfaction with treatment of breast cancer: does surgeon specialization matter? J Clin Oncol 2007;25:3694-8.
6. Sclafani LM, BleznakA, Kelly T, El-Tamer MB. Training a new generation of breast surgeons: are we succeeding? Ann Surg Oncol 2012;19:1856-61.

7. Maxwell J, Roberts A, Cil T, Somogi R, Osman F. Current practices and barriers to the integration of oncoplastic breast surgery: a Canadian perspective. Ann Surg Oncol 2016;23:3259-65.

8. Society of Surgical Oncology (sso). SSO-Approved Breast Oncology Fellowship Training Programs [Web page]. Rosemont, IL: sso; n.d. [Current version available at: http://www. surgonc.org/training-fellows/fellows-education/breastoncology/program-list; cited 27 September 2016]

9. Baildam A, Bishop H, Boland G, et al. on behalf of the Association of Breast Surgery at BAso, the Association of Plastic Surgery at BAPRAS, and the Training Interface Group in Breast Surgery. Oncoplastic breast surgery - a guide to good practice. Eur JSurg Oncol 2007;33(suppl 1):S1-23. [Erratum in: Eur J Surg Oncol 2008;34:II]

10. Cardoso MJ, Macmillan RD, MerckB, MunhozAM, Rainsbury R. Training in oncoplastic surgery: an international consensus. The 7th Portuguese Senology Congress, Vilamoura, 2009. Breast 2010;19:538-40. 
11. Zucca Matthes AG, Viera RA, Michelli RA, et al. The development of an oncoplastic training center-отс. Int J Surg 2012;10:265-9.

12. Rainsbury RM. Training and skills for breast surgeons in the new millennium. ANZ J Surg 2003;73:511-16.

13. Down SK, Pereira JH, Leinster S, Simpson A. Training the oncoplastic breast surgeon-current and future perspectives. Gland Surg 2013;2:126-7.

14. Yunaev M, Hingston G. Oncoplastic breast surgery: a regional Australian 2012 fellowship experience. ANZ J Surg 2013;83:624-9.

15. Yunaev M, Hingston G. Oncoplastic breast surgery in Australia and New Zealand: 2014 and beyond. Gland Surg 2014;3:77-80.

16. Clough KB, Kaufman GJ, Nos C, Buccimazza I, Sarfati IM. Improving breast cancer surgery: a classification and quadrant per quadrant atlas for oncoplastic surgery. Ann Surg Oncol 2010;17:1375-91.

17. Cataliotti L, De Wolf C, Holland R, et al. Guidelines on the standards of training of specialised health professionals dealing with breast cancer. Eur J Cancer 2007;43:660-75.

18. Sneeuw KC, Aaronson NK, Yarnold JR, et al. Cosmetic and functional outcomes of breast conserving treatment for early stage breast cancer. 2 . Relationship with psychosocial functioning. Radiother Oncol 1992;25:160-6.

19. Al-Ghazal SK, Fallowfield L, Blamey RW. Does cosmetic outcome from treatment of primary breast cancer influence psychosocial morbidity? Eur J Surg Oncol 1999;25:571-3.

20. Waljee JF, Hu ES, Ubel PA, Smith DM, Newman LA, Alderman AK. Effect of esthetic outcome after breast-conserving surgery on psychosocial functioning and quality oflife. JClin Oncol 2008;26:3331-7. 